\title{
Smoothing Parameter Optimization Routine for High-Quality a Priori Estimates in Forecasting-Aided State Estimation
}

\author{
Md Rejwanur R. Mojumdar, José M. Cano, Shahid Jaman, and Gonzalo A. Orcajo \\ Department of Electrical Engineering \\ University of Oviedo \\ 33204 Gijón, Spain \\ mojumdarrejwanur.uo@uniovi.es; jmcano@uniovi.es; shahid.jaman.aust@gmail.com; gonzalo@uniovi.es
}

\begin{abstract}
Forecasting-aided state estimation is a topic of great research interest for future intelligent power grids. Estimators in this class commonly use an exponential smoothing based forecasting model to provide $a$ priori estimates of the states. This forecasting capability is advantageous to the system operators in taking proper actions. In this work, authors propose a periodical or event-triggered routine to improve the output of this class of estimators. This routine will use an efficient optimization scheme to obtain custom smoothing parameters for each of the state variables in the forecasting model. The authors include a case study to demonstrate the high quality of the $a$ priori estimates obtained with the proposed method.
\end{abstract}

Index Terms-A priori estimates, Forecasting-aided state estimation, intelligent power grid, linear exponential smoothing.

\section{INTRODUCTION}

In the seminal work of Debs and Larson [1], they proposed the very fundamental algorithm for Dynamic State Estimation (DSE). Any serious attempt to model the time behavior of the system state was avoided, assuming a simple dynamic linear model where the parameters are either identity matrices or zeros. Then, at their pioneering work, Schweppe and Masiello [2] introduced another algorithm for tracking the states. It proposes a simultaneous estimation of states and parameters, which failed to identify dynamic patterns properly. It is important to note that the term "dynamic" may be ambiguous, as power system dynamics is strongly associated with the stability concept and the oscillatory response of the system during transients. Semantic arguments in the State Estimation (SE) context have led researchers to coin the term Forecastingaided State Estimation (FASE) [3], and thus, in this work, it is described with that denotation.

FASE, which can be seen as an estimated state assisted by prediction, has gained significant research interest as the power sector is undergoing a profound change. The emergence of Smart Grid (SG), Distributed Generation (DG),

This work was supported by the Spanish Ministry of Economy and Competitiveness through the National Plan for Scientific and Technical Research and Innovation under grant ENE2014-52272-R. and Demand Response (DR) have posted crucial questions on power system paradigm. Though Weighted Least Squares (WLS) SE is a well-established and mature technique for transmission systems and also offers some recent solutions for three-phase unbalanced distribution systems [4], FASE appears to have certain advantages for the operation, control, and protection of future Intelligent Distribution Networks (IDNs). While traditional WLS based algorithms, perform the SE process from a fixed image of the network obtained at each scan of the measurements, FASE includes the effect of past measurements in its estimation. Importantly, FASE allows an a priori estimation of the future state of the system for the next time steps, which is particularly useful in broadening the scope of the operators or the Energy Management System (EMS), because some functions, like economic dispatching and security assessment can be performed in advance.

In FASE, a prediction stage anticipates future values of the state variables assuming a forecasting model of the system. Some parameters of this model need to be identified. The forecasting model used to be tedious, costly and full of uncertainties. A major progress in FASE comes from the work of [5], which provides two dynamic state transition models, both capable of following system changes. The dynamic model using Linear Exponential Smoothing (LES) [6] to predict the forecasting model parameters, and consequently, the a priori estimates, was found better for forecasting the states. Once $a$ priori estimates are available, FASE proceeds with a filtering stage using techniques such as the Extended Kalman Filter (EKF), Iterated Kalman filter (IKF), Unscented Kalman Filter (UKF), or Particle Filter. This work, focuses and contributes to the improvement of a priori estimations, i.e., the prediction stage. Many of the high-impact FASE related research works, such as [3], [7]-[9], have used LES to identify the forecasting model parameters, and thus, LES is also taken as the basis for the present proposal.

In this work, the authors propose and implement an efficient optimization routine to tune up customized parameters for LES in a particular power system. The routine can be executed in either an event-triggered or periodical basis, allowing a desirable 
adaptation of the forecasting parameters to topological changes or seasonal variations. FASE can benefit from high-quality a priori estimates in different ways. Firstly, high-quality $a$ priori estimates can be useful for event-triggered Distribution System State Estimation (DSSE) or Multi-Area State Estimation (MASE) techniques, such as [10]. High-quality a priori estimates can also be useful for some topology identification algorithms, such as [11]. Finally, the availability of improved a priori estimates, reduces the number of iterations needed during the filtering stage, thus improving the efficiency of FASE techniques.

For the benefit of the reader, a traditional formulation of the mathematical model used in FASE, [5], is presented in Section II. Section III introduces the proposed optimization method, capable of providing customized exponential smoothing parameters for each state variable. In Section IV, a case study is presented to illustrate the benefits of the proposal. Finally, Section V summarizes the most important results of this study.

\section{The Mathematical Model}

Four parts can be distinguished in FASE: the measurement model, the dynamic forecasting model, the identification of forecasting parameters, and the state filtering process [5]. They are briefly described in this section, with special attention given to the forecasting stage in which this proposal is focused.

\section{A. The Measurement Model}

The measurement model is essential for the filtering stage. At an instant of time, $k$, the measurement vector, $\mathbf{z}_{\mathbf{k}}$, of $m$ actual observations is related with the state vector, $\mathbf{x}_{\mathbf{k}}$, containing $n$ state variables as

$$
\mathbf{z}_{\mathbf{k}}=\mathbf{h}\left(\mathbf{x}_{\mathbf{k}}\right)+\mathbf{e}_{\mathbf{k}},
$$

where, $\mathbf{h}$ is a vector containing nonlinear functions, based on Ohm's and Kirchhoff's laws, which transforms the state vector space to the theoretical measurement space. The measurement error vector, $\mathbf{e}_{\mathbf{k}}$, contains white Gaussian noise with zero mean and a covariance matrix $\mathbf{R}_{\mathbf{k}}$ for weighing inputs with their estimated precision.

It is assumed that $m>n$, and there is a set of $n$ equations in $\mathbf{h}$ which are independent. By linearizing around an operating point $\mathbf{x}_{\mathbf{k}}^{0}$, (1) can be rewritten as

$$
z_{k}=H_{k} \boldsymbol{x}_{k}+\mathbf{E}_{\mathbf{k}},
$$

where, $z_{k}=\mathbf{z}_{\mathbf{k}}-\mathbf{h}\left(\mathbf{x}_{\mathbf{k}}^{0}\right), \boldsymbol{x}_{\boldsymbol{k}}=\mathbf{x}_{\mathbf{k}}-\mathbf{x}_{\mathbf{k}}^{0}$ and $\boldsymbol{H}_{\boldsymbol{k}}=\left.\frac{\partial \mathbf{h}}{\partial \mathbf{x}}\right|_{x=\mathbf{x}_{\mathbf{k}}^{0}}$. $\mathbf{E}_{\mathbf{k}}$ is white Gaussian noise with zero mean that represents the combined error of the measurement and linearization process.

\section{B. Dynamic Forecasting Model}

It is assumed that the state vector complies with the following dynamic model:

$$
\mathbf{x}_{\mathbf{k}+1}=\mathbf{F}_{\mathbf{k}} \mathbf{x}_{\mathbf{k}}+\mathbf{G}_{\mathbf{k}}+\mathbf{w}_{\mathbf{k}},
$$

where $\mathbf{F}_{\mathbf{k}}$ is a non-zero $(n \times n)$ diagonal matrix, $\mathbf{G}_{\mathbf{k}}$ is a nonzero $(n \times 1)$ vector and $\mathbf{w}_{\mathbf{k}}$ is white Gaussian noise with zero mean and covariance matrix $\mathbf{Q}_{\mathbf{k}}$.

\section{Identification of Forecasting Parameters}

In this step, the parameters of the dynamic forecasting model, $\mathbf{F}_{\mathbf{k}}$ and $\mathbf{G}_{\mathbf{k}}$, are derived. The quality of this identification determines the goodness of the states' forecast. LES is traditionally used in FASE for this task. From the taxonomy of exponential smoothing methods [6], which consists of 15 models, those with additive trend and no seasonal component have been widely adopted in FASE implementations. In this work the Damped Trend Method (DTM) is used, but the proposed methodology can be applied to other models in a straightforward manner. DTM is a damped variant of the popular Holt's method which improves its long-term forecasting features. The equations for the DTM can be formulated for each state variable, $i$, and sample, $k$, as,

$$
\begin{aligned}
& \text { Level: } & l_{k}^{i} & =\alpha^{i} \hat{x}_{k}^{i}+\left(1-\alpha^{i}\right)\left(l_{k-1}^{i}+\phi^{i} b_{k-1}^{i}\right), \\
& \text { Trend: } & b_{k}^{i} & =\beta^{i}\left(l_{k}^{i}-l_{k-1}^{i}\right)+\left(1-\beta^{i}\right) \phi^{i} b_{k-1}^{i}, \\
& \text { Forecast: } & \widetilde{x}_{k+1}^{i} & =l_{k}^{i}+\phi^{i} b_{k}^{i},
\end{aligned}
$$

where $\alpha^{i}, \beta^{i}$ and $\phi^{i}$ are the smoothing parameters and dampen factor, respectively. $l_{k}^{i}$ and $b_{k}^{i}$ are the level and trend, $\hat{x}_{k}^{i}$ is the a posteriori estimate and $\widetilde{x}_{k+1}^{i}$ is the a priori estimate. The backcasting method can be used for initialization purposes.

Taking the mathematical expectation of $\mathbf{x}_{\mathbf{k}+\mathbf{1}}$ in (3) as the forecast value, the a priori estimate can be expressed as

$$
\widetilde{\mathbf{x}}_{\mathbf{k}+\mathbf{1}}=\mathbf{F}_{\mathbf{k}} \hat{\mathbf{x}}_{\mathbf{k}}+\mathbf{G}_{\mathbf{k}} .
$$

Identifying terms in (7) and (4)-(6) leads to

$$
\begin{aligned}
F_{k}^{i i} & =\alpha^{i}\left(1+\phi^{i} \beta^{i}\right) \\
G_{k}^{i} & =\left(1+\phi^{i} \beta^{i}\right)\left(1-\alpha^{i}\right) \widetilde{x}_{k}^{i}-\phi^{i} \beta^{i} l_{k-1}^{i}+\phi^{i 2}\left(1-\beta^{i}\right) b_{k-1}^{i} .
\end{aligned}
$$

\section{State Filtering}

The objective of the filtering stage is to find an a posteriori estimation of the system state. This is done by minimizing the root mean square error between theoretical observations and actual observations, for which different techniques have been proposed. State filtering is out of the scope of this work, however, it is worth mentioning that the iterated extended Kalman filter [5] was used while conducting the case studies for the present proposal.

\section{SMOothing PARAmeter Optimization}

Traditional works on FASE with LES use preassigned uniform values as smoothing parameters for the state variables, e.g. $\alpha^{\text {all }}=0.6, \beta^{\text {all }}=0.4$ and $\phi^{\text {all }}=0.95$. These figures are selected based on the experience of the user or at most, after some trial and error. In this section, an efficient process for the optimization of these parameters, which provides custom values for each state variable, is proposed.

The load profile of electric networks shows clear seasonality patterns, as is the case of a daily pattern. This fact can be exploited in order to optimize the smoothing parameters of each state variable given a set of daily measurements, so as to obtain improved estimates in the future. The algorithm proposed in this section can be scheduled periodically or 
executed on an event-triggered basis in order to obtain a better adjustment of the estimates to long-term seasonality patterns, such as monthly pattern, or topology changes.

The accuracy of a priori estimates of the state variables should be assessed by comparing them with the "exact" values of that states. Unfortunately, in real applications those "exact" values are unknown, so they have to be compared with $a$ posteriori estimates. With the goal of error minimization, different accuracy indicators have been proposed, such as Mean Absolute Error (MAE), Mean Squared Error (MSE), Root Mean Squared Error (RMSE), Mean Absolute Percentage Error (MAPE) or Mean Absolute Scaled Error (MASE) [6]. In this work RMSE is used, which is particularly suitable for exponential smoothing, as proportionally larger weight is put in larger errors. RMSE for a specific state variable, $i$, can be defined as

$$
R M S E^{i}=\sqrt{\frac{1}{K} \sum_{k=1}^{K}\left(\widetilde{x}_{k}^{i}-\hat{x}_{k}^{i}\right)^{2}},
$$

where, $K$ is the total number of time steps considered in the calculation (e.g. 96 for a daily profile with quarter-hourly measurements).

The optimization problem to be solved can be expressed as the minimization of an aggregate of those $R M S E^{i}$ values. The search space for this optimization task grows exponentially with the number of state variables, which can make the problem infeasible for medium or large systems. This is caused by the fact that $R M S E^{j}$ depends not only on $\alpha^{j}, \beta^{j}$ and $\phi^{j}$, but also on the parameters for the rest of the state variables, i.e. $\alpha^{i}, \beta^{i}$ and $\phi^{i}$ with $i \neq j$, through their influence in the $a$ posteriori estimates. In order to get rid of this difficulty, the present proposal makes use of the low weight of this second factor of influence, which allows a decoupled calculation of the smoothing parameters of each state variable, provided that a posteriori estimates are iteratively corrected through an external loop (two or three iterations suffice in most cases). With this solution, which is presented in Algorithm 1, the optimization process can be run in a time with a linear dependency on the number of state variables, making it valid for large networks. Particle Swarm Optimization (PSO) is used in this work as an optimization tool, though a deterministic procedure could also be employed for the same purpose.

The embedded PSO algorithm solves the following constrained objective function for each of the state variables in a decoupled way,

$$
\begin{aligned}
\operatorname{minimize} & R M S E^{i}=f\left(\alpha^{i}, \beta^{i}, \phi^{i}\right) \\
\text { subject to } & 0<\alpha^{i}<2 \\
& 0<\beta^{i}<4 / \alpha^{i}-2 \\
& 0<\phi^{i}<1
\end{aligned}
$$

The traditional bounds of the smoothing parameters, i.e. $0<$ $\alpha<1,0<\beta<\alpha$, based on an interpretation as a weighted average of past values, has been superseded by the state space approach of LES, in which the limits shown in (9) are based

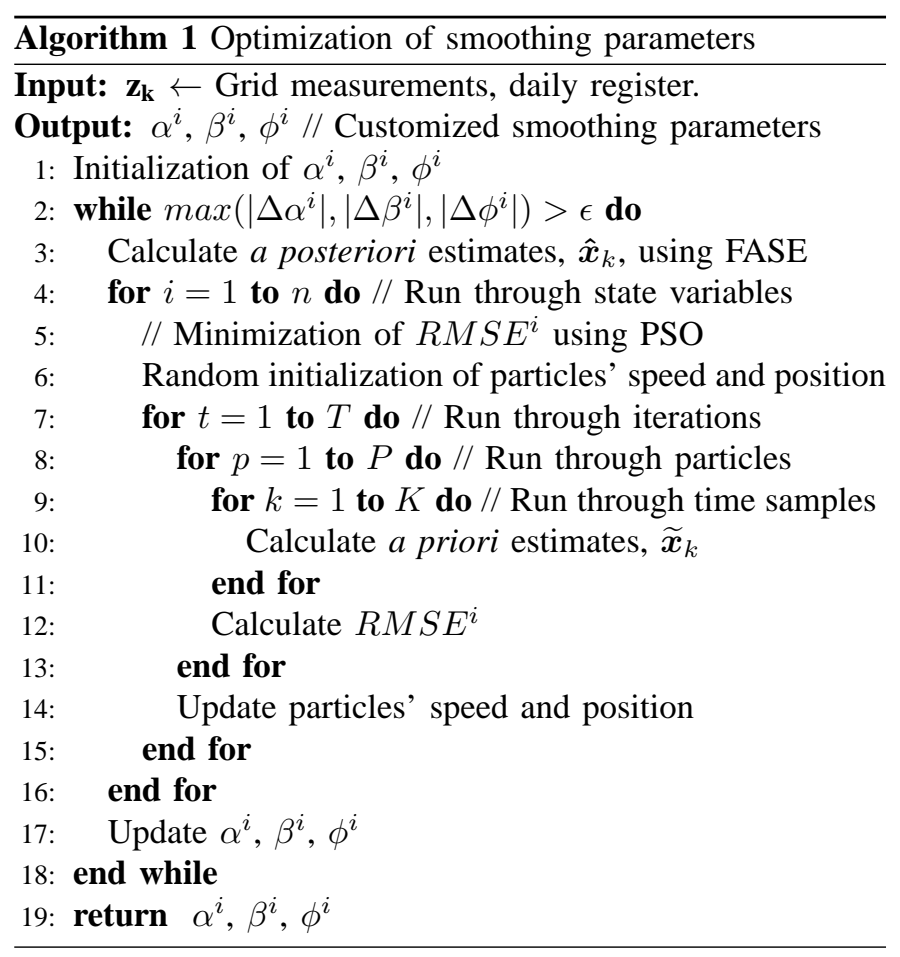

on a stability argument [6]. Solutions out of these bounds are rejected within the PSO algorithm by adding a penalization function.

Within the PSO algorithm [12], a set of $P$ particles explores the domain of solutions. Each of these entities, $p$, is characterized by a position, $y_{p}$, i.e. a set of $\alpha^{i}, \beta^{i}$ and $\phi^{i}$ values, and a speed, $v_{p}$, in said domain, where they are randomly located at initialization. At each iteration, $t$, the fitness of each particle is assessed according to the evaluation of the objective function (9), and the best position for each particle, $y_{b}{ }_{p}^{t}$, and for the whole swarm, $y_{s w}{ }^{t}$, are stored. Then, the speed of each particle is updated as

$$
v_{p}^{t+1}=\omega v_{p}^{t}+c_{1} r_{1}^{t}\left(y_{b}^{t}-y_{p}^{t}\right)+c_{2} r_{2}^{t}\left(y_{s w}^{t}-y_{p}^{t}\right),
$$

where $r_{1}$ and $r_{2}$ are random numbers in the range $[0,1)$ used to preserve the diversity of the population, and $\omega$ is a constant, called inertia weight, that determines the influence of the preceding speed value. Finally, the effect on the new speed of both the best result obtained by each particle and the whole swarm is determined by constants $c_{1}$ and $c_{2}$, usually known as cognitive and social weights, respectively. From (10), a newly updated position for each particle is calculated as

$$
\begin{aligned}
& y_{p}^{t+1}=y_{p}^{t}+v_{p}^{t+1} . \\
& \text { IV. CASE STUdy }
\end{aligned}
$$

A well-tested industrial power system previously used in [12] has been adopted to demonstrate the usefulness of the proposal. The said system, depicted in Fig. 1, is a 9-bus simplified version of the industrial network of a steel mill in the north of Spain. The specific data of this network, together with the set points of the embedded tapped transformers, 


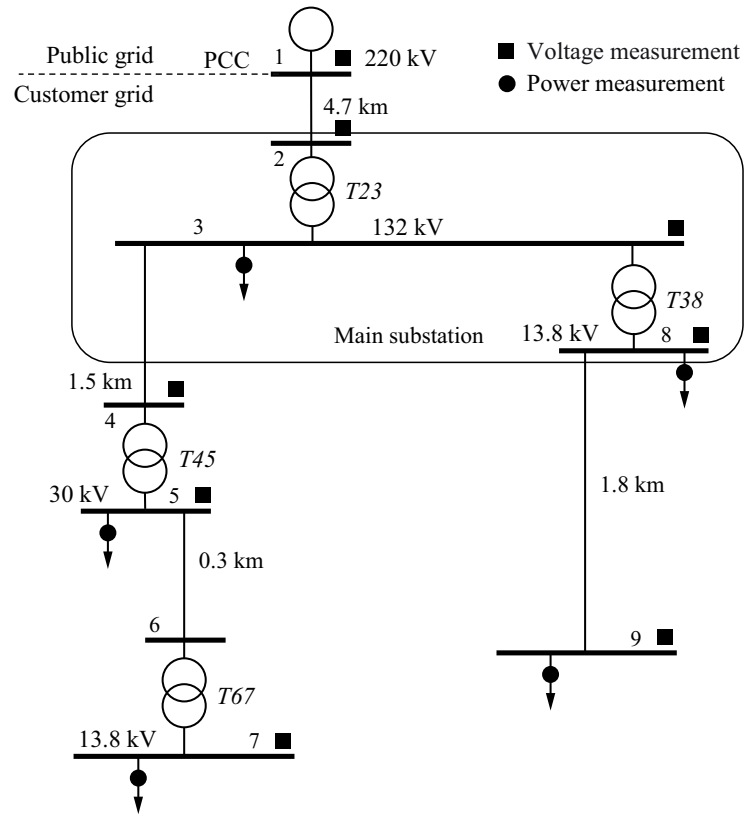

Fig. 1. 9-bus Industrial distribution grid

have been summarized in Table I. The implementation of the algorithms used in this case study was carried out in the MATLAB programming platform and tested in an Intel Core i5 - 6400M - CPU $2.70 \mathrm{GHz}$ computer.

TABLE I

PARAMETERS AND SETUP OF THE DistribUtion GRID

\begin{tabular}{|c|c|c|c|c|}
\hline \multicolumn{5}{|c|}{ Lines (z) and transformers $\left(S_{n}, R_{s c}, X_{s c}, \operatorname{tap}\right)$} \\
\hline Lin & 40i $\Omega / \mathrm{km}$ & T23 $2 \times 270$ MVA & $0.90 \%$ & $12.97 \% \quad 0.98$ \\
\hline & 1i $\Omega / \mathrm{km}$ & T45 $3 \times 37.5$ MVA & $0.90 \%$ & $8.95 \% \quad 0.99$ \\
\hline $\mathrm{Li}$ & $0.568+0.133 \mathrm{i} \Omega / \mathrm{km}$ & $10 \mathrm{MVA}$ & $0.95 \%$ & $4.76 \% \quad 1.00$ \\
\hline & $0.161+0.112 \mathrm{i} \Omega / \mathrm{km}$ & T38 $3 \times 50 \mathrm{MVA}$ & $0.92 \%$ & $\begin{array}{lll}7.95 \% & 0.98\end{array}$ \\
\hline \multicolumn{5}{|c|}{ Loads (P, Q - daily peak values) } \\
\hline Bus 3 & $\mathrm{~W}, \quad 56.8 \mathrm{Mv}$ & Bus & $42.0 \mathrm{~N}$ & 47.8 Mvar \\
\hline & $24.0 \mathrm{MW}, 21.9 \mathrm{Mva}$ & Bus 9 & & \\
\hline Bus 7 & $7.0 \mathrm{MW}$ & & & \\
\hline
\end{tabular}

A set of measurements, in the minimum number to make the system observable, were taken as true values in order to allow for the calculation of "exact" results for the state of the system. Notice, that this is only done with the aim of validating the accuracy of the proposed a priori estimates, i.e., these results are not used during the optimization process. The daily load profile at the different buses of the grid, using quarterhourly real and reactive power values, is considered. A realistic performance was obtained by making use of the ADRESCONCEPT database [13]. Table I shows the peak values of these load profiles. The state of the system is obtained from these data through the Direct Approach power flow algorithm [14], which is specially suited for radial grids. Gaussian noise was added to an augmented set of measurements, which are highlighted in Fig. 1, to obtain corrupted values valid to feed the FASE algorithm is a realistic fashion: $0.1 \%$ and $2.0 \%$ of the mean of the normal distribution was selected as the standard deviation of voltages and power injections, respectively. Thus, these standard deviations were also used for the error covariance matrix $\mathbf{R}$ in the FASE algorithm.

By following the method described in Section III, optimum values of $\alpha^{i}, \beta^{i}$ and $\phi^{i}$ were calculated for the 17 state variables of the grid. These values are shown in Table II. The good adjustment of these parameters to the optimization data set can be observed in Table III. Here, the RMSE value is calculated for the a priori estimates in three different cases: (a) the naïve case, in which the state of every variable is forecast using the value of the preceding state, i.e $\widetilde{x}_{k+1}^{i}=\hat{x}_{k}^{i}$, (b) a case with non-optimized parameters, taken identically for all the state variables, and (c) the proposed improvement, which uses custom optimized parameters. This last case, clearly beats (a) and (b). In Table III, $R M S E$ was calculated considering the error between the a priori estimates and the "exact" results, not to let a posteriori estimates hide any estimation errors.

TABLE II

OPTIMUM $\alpha^{i}, \beta^{i}$ AND $\phi^{i}$ FOR STATE FORECASTING

\begin{tabular}{cccccccc}
\hline Voltage & $\alpha^{i}$ & $\beta^{i}$ & $\phi^{i}$ & Phase & $\alpha^{i}$ & $\beta^{i}$ & $\phi^{i}$ \\
\hline \hline$V_{1}$ & 0.157 & 0.430 & 0.000 & $\theta_{1}$ & - & - & - \\
$V_{2}$ & 0.643 & 0.759 & 0.000 & $\theta_{2}$ & 0.825 & 2.850 & 0.329 \\
$V_{3}$ & 1.208 & 1.311 & 0.356 & $\theta_{3}$ & 0.845 & 2.733 & 0.335 \\
$V_{4}$ & 1.206 & 1.317 & 0.360 & $\theta_{4}$ & 0.852 & 2.694 & 0.332 \\
$V_{5}$ & 1.153 & 1.470 & 0.461 & $\theta_{5}$ & 0.916 & 2.367 & 0.326 \\
$V_{6}$ & 1.127 & 1.550 & 0.457 & $\theta_{6}$ & 0.908 & 2.407 & 0.315 \\
$V_{7}$ & 1.217 & 1.287 & 0.472 & $\theta_{7}$ & 0.867 & 2.613 & 0.298 \\
$V_{8}$ & 1.132 & 1.533 & 0.397 & $\theta_{8}$ & 0.730 & 3.478 & 0.330 \\
$V_{9}$ & 1.151 & 1.477 & 0.417 & $\theta_{9}$ & 0.782 & 3.116 & 0.317 \\
\hline
\end{tabular}

TABLE III

$R M S E$ (IN THOUSANDS) FOR A PRIORI ESTIMATES OF STATE VARIABLES APPLIED TO THE OPTIMIZATION DATA SET

\begin{tabular}{cccccccc}
\hline & \multicolumn{3}{c}{ Case } & & \multicolumn{3}{c}{ Case } \\
Voltage & (a) & (b) & (c) & Phase & (a) & (b) & (c) \\
\hline \hline$V_{1}$ & 0.796 & 0.792 & 0.681 & $\theta_{1}$ & - & - & - \\
$V_{2}$ & 0.828 & 0.843 & 0.792 & $\theta_{2}$ & 0.106 & 0.129 & 0.085 \\
$V_{3}$ & 1.859 & 2.265 & 1.457 & $\theta_{3}$ & 1.486 & 1.661 & 1.265 \\
$V_{4}$ & 1.887 & 2.298 & 1.473 & $\theta_{4}$ & 1.490 & 1.665 & 1.269 \\
$V_{5}$ & 3.052 & 3.773 & 2.155 & $\theta_{5}$ & 2.341 & 2.651 & 1.957 \\
$V_{6}$ & 3.102 & 3.831 & 2.194 & $\theta_{6}$ & 2.325 & 2.631 & 1.967 \\
$V_{7}$ & 4.879 & 5.998 & 3.430 & $\theta_{7}$ & 3.436 & 3.896 & 2.874 \\
$V_{8}$ & 3.553 & 4.399 & 2.699 & $\theta_{8}$ & 2.590 & 2.871 & 2.236 \\
$V_{9}$ & 3.873 & 4.744 & 2.874 & $\theta_{9}$ & 2.647 & 3.003 & 2.297 \\
\hline
\end{tabular}

However, the applicability of the method is not still demonstrated until this point, as the optimization process is based in past values from an optimization data set. To probe the real benefit of the proposal, a test data set of power injections was extracted from the ADRES-CONCEPT database. This set is built with data from the same aggregated loads used in the optimization data set, but corresponding to different days. The risk of overfitting is avoided due to clearly diverse profiles, as can be seen in Fig. 2, where the measurements for real and reactive power at bus 8 has been depicted both for the optimization and test data sets. The FASE algorithm was run again on this new test data set for the three cases described 
above, but using the previously optimized $\alpha^{i}, \beta^{i}$ and $\phi^{i}$ values of case (c). Table IV clearly shows that the daily seasonality of the measurements leads to improved a priori estimates also for future values. In order to further illustrate the interest of the proposed method, Fig. 3 shows the daily profile for the voltage at node 9 and for the phase angle at node 6 . In this figure, the true values of these state variable are depicted together with the a priori estimates from cases (a), (b) and (c). The a posteriori estimates of the different cases are not shown in the figure for the sake of clarity.

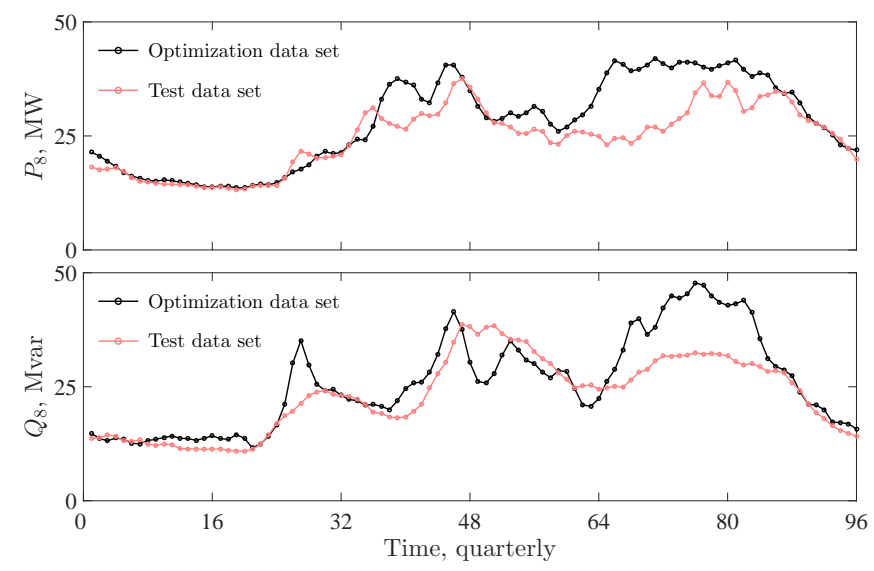

Fig. 2. Daily load profile from measurements of power injections at bus 8: optimization and test data sets

TABLE IV

$R M S E$ (IN THOUSANDS) FOR A PRIORI ESTIMATES OF STATE VARIABLES APPLIED TO THE TEST DATA SET

\begin{tabular}{cccccccc}
\hline & \multicolumn{3}{c}{ Case } & & \multicolumn{3}{c}{ Case } \\
Voltage & (a) & (b) & (c) & Phase & (a) & (b) & (c) \\
\hline \hline$V_{1}$ & 0.733 & 0.726 & 0.620 & $\theta_{1}$ & - & - & - \\
$V_{2}$ & 0.743 & 0.743 & 0.698 & $\theta_{2}$ & 0.114 & 0.143 & 0.104 \\
$V_{3}$ & 1.181 & 1.292 & 1.007 & $\theta_{3}$ & 1.496 & 1.750 & 1.367 \\
$V_{4}$ & 1.199 & 1.314 & 1.017 & $\theta_{4}$ & 1.504 & 1.761 & 1.375 \\
$V_{5}$ & 1.933 & 2.215 & 1.478 & $\theta_{5}$ & 2.366 & 2.817 & 2.130 \\
$V_{6}$ & 1.968 & 2.259 & 1.490 & $\theta_{6}$ & 2.365 & 2.811 & 2.141 \\
$V_{7}$ & 3.038 & 3.553 & 2.249 & $\theta_{7}$ & 3.462 & 4.315 & 3.049 \\
$V_{8}$ & 2.103 & 2.211 & 1.634 & $\theta_{8}$ & 2.524 & 2.915 & 2.350 \\
$V_{9}$ & 2.408 & 2.567 & 1.828 & $\theta_{9}$ & 2.615 & 3.058 & 2.456 \\
\hline
\end{tabular}

\section{Conclusions}

High-quality a priori estimates can help in the implementation of smart grid systems in many ways. This work presents a straightforward method to formulate improved a priori estimates in FASE. The proposed methodology uses PSO as a tool to optimize LES parameters. This contribution clearly demonstrates that the use of custom optimized parameters for each of the state variables overcomes the traditional use of uniform parameters in FASE applications. This type of optimization can successfully exploit the seasonality pattern of load profiles on daily basis. The execution time of the proposed optimization method grows linearly with the number of state variables, thus making it suitable to be applied to large grids.

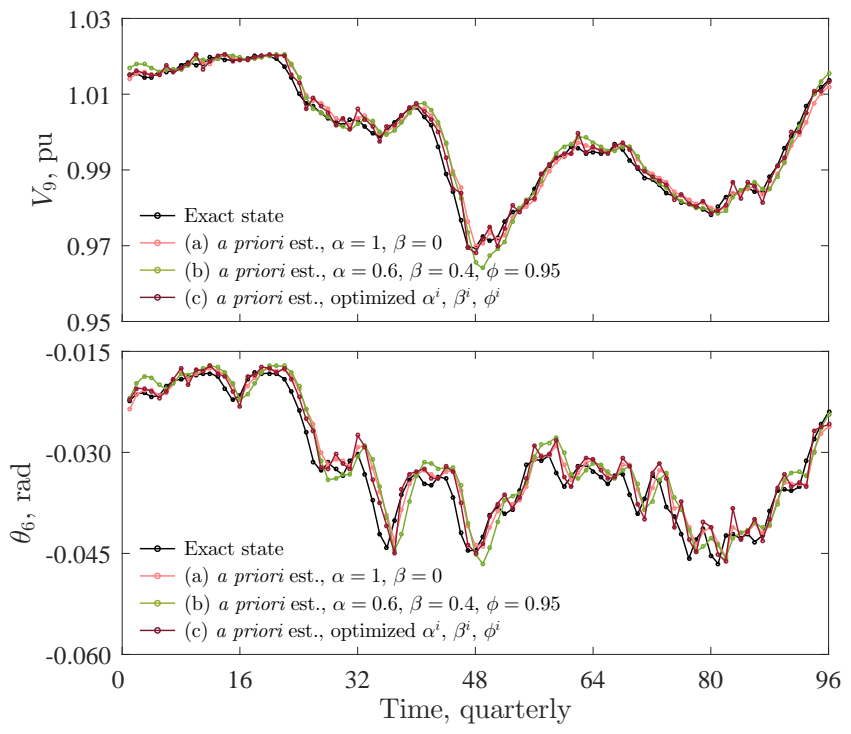

Fig. 3. A priori estimates for voltage at bus 9 and phase angle at bus 6 . Exact state, (a) naïve case, (b) uniform values, (c) optimized parameters

\section{REFERENCES}

[1] A. S. Debs and R. E. Larson, "A dynamic estimator for tracking the state of a power system," IEEE Transactions on Power Apparatus and Systems, vol. PAS-89, no. 7, pp. 1670-1678, Sept 1970.

[2] F. C. Schweppe and R. D. Masiello, "A tracking static state estimator," IEEE Transactions on Power Apparatus and Systems, vol. PAS-90, no. 3, pp. 1025-1033, May 1971.

[3] M. B. Do Coutto Filho and J. C. S. de Souza, "Forecasting-aided state estimation - Part I: Panorama," IEEE Transactions on Power Systems, vol. 24, no. 4, pp. 1667-1677, 2009.

[4] F. Ahmad, A. Rasool, E. Ozsoy, S. Rajasekar, A. Sabanovic, and M. Elitaş, "Distribution system state estimation - a step towards smart grid," Renewable and Sustainable Energy Reviews, 2017.

[5] A. L. da Silva, M. Do Coutto Filho, and J. de Queiroz, "State forecasting in electric power systems," in IEE Proceedings C (Generation, Transmission and Distribution), vol. 130, no. 5, 1983, pp. 237-244.

[6] R. J. Hyndman, A. B. Koehler, J. K. Ord, and R. D. Snyder, Forecasting with Exponential Smoothing. The State Space Approach. SpringerVerlag, 2008.

[7] K.-R. Shih and S.-J. Huang, "Application of a robust algorithm for dynamic state estimation of a power system," IEEE Transactions on Power Systems, vol. 17, no. 1, pp. 141-147, 2002.

[8] J.-M. Lin, S.-J. Huang, and K.-R. Shih, "Application of sliding surfaceenhanced fuzzy control for dynamic state estimation of a power system," IEEE Transactions on Power Systems, vol. 18, no. 2, pp. 570-577, 2003.

[9] S.-J. Huang and K.-R. Shih, "Dynamic-state-estimation scheme including nonlinear measurement function considerations," IEE Proc. Generation, Transmission and Dist., vol. 149, no. 6, pp. 673-678, 2002.

[10] R. C. Francy, A. M. Farid, and K. Youcef-Toumi, "Event triggered state estimation techniques for power systems with integrated variable energy resources," ISA Transactions, vol. 56, pp. 165-172, 2015.

[11] R. Singh, E. Manitsas, B. C. Pal, and G. Strbac, "A recursive Bayesian approach for identification of network configuration changes in distribution system state estimation," IEEE Transactions on Power Systems, vol. 25 , no. 3, pp. 1329-1336, 2010.

[12] J. M. Cano, J. G. Norniella, C. H. Rojas, G. A. Orcajo, and J. Jatskevich, "Application of loop power flow controllers for power demand optimization at industrial customer sites," in 2015 IEEE Power Energy Society General Meeting, July 2015, pp. 1-5.

[13] ADRES-CONCEPT, "EZ-IF: Development of concepts for ADRES autonomous decentralized regenerative energy systems, project no. 815674 . Funded by the Austrian climate and energy fund and performed under the program Energie der Zukunft." 2010.

[14] J.-H. Teng, "A direct approach for distribution system load flow solutions," IEEE Trans. on Power Del., vol. 18, no. 3, pp. 882-887, 2003. 